\title{
Convergence in the Physical Appearance of Spouses ${ }^{1}$
}

\author{
R. B. Zajonc, ${ }^{2}$ Pamela K. Adelmann, Sheila T. Murphy, and \\ Paula M. Niedenthal \\ University of Michigan
}

This study attempted to determine whether people who live with each other for a long period of time grow physically similar in their facial features. Photographs of couples when they were first married and 25 years later were judged for physical similarity and for the likelihood that they were married. The results showed that there is indeed an increase in apparent similarity after 25 years of cohabitation. Moreover, increase in resemblance was associated with greater reported marital happiness. Among the explanations of this phenomenon that were examined, one based on a theory of emotional efference emerged as promising. This theory proposes that emotional processes produce vascular changes that are, in part, regulated by facial musculature. The facial muscles are said to act as ligatures on veins and arteries, and they thereby are able to divert blood from, or direct blood to, the brain. An implication of the vascular theory of emotional efference is that habitual use of facial musculature may permanently affect the physical features of the face. The implication holds further that two people who live with each other for a longer period of time, by virtue of repeated empathic mimicry, would grow physically similar in their facial features. Kin resemblance, therefore, may not be simply a matter of common genes but also a matter of prolonged social contact.

Dear Dr. Brothers: I am a 17-year-old who looks at all the couples passing by. Old married couples, people in love-or at least who seem to be in love. I am struck by the fact that they almost always look alike. I heard that opposites attract. . . .

${ }^{1}$ This work was supported by Grant BNS-8505981 from the National Science Foundation. We wish to thank all those who offered their photographs for the purposes of this study, and Joseph R. Zajonc for his helpful suggestions.

${ }^{2}$ Address all correspondence to R. B. Zajonc, Research Center for Group Dynamics, The University of Michigan, Ann Arbor, Michigan 48106. 
Dear A. L.: As far as physical appearance is concerned, likes seem to attract. Some experts feel that this resemblance may partly be explained by the fact that couples who've lived together for some time usually eat the same diet and share the same habits.

The Joyce Brothers Column April 1985

It is commonly believed that people who select each other as mates are more similar in appearance than randomly chosen couples. This belief has considerable support in the empirical literature. Rushton, Russell and Wells (1985), for example, aggregated a variety of correlations between spouses' physical characteristics and found consistently positive coefficients for height, chest breadth, length of the earlobe, minimum neck circumference, middle finger length, and several others. However, independently of their physical similarity at marriage, spouses are believed to grow physically more similar as they grow older. Evidence for this conjecture does not exist and, moreover, it isn't at all clear why the conjecture should be true.

This study seeks to determine whether the faces of spouses appear more similar after 25 years of marriage. If the suspected convergence in the physical appearance of spouses can be demonstrated, then we will consider the question of possible causes and mechanisms. Among the possible explanations we shall pay particular attention to is a theory of emotional efference recently reclaimed (Zajonc, 1985) - a theory that has as one implication that repeated patterns of emotional expression may produce slight permanent changes in the facial features, and that by virtue of prolonged mutual mimicry two people living in close proximity might grow similar in these patterns.

\section{METHOD}

\section{Overview}

Twenty-four photographs of men and 24 photographs of women were judged for their resemblance to each other, and for the likelihood of a given man and a given women being married to each other. The photographs were actually those of the partners of 12 married couples. Half of the photographs were taken at the time of the men's and women's wedding, and half about 25 years later.

\section{Subjects}

A total of 110 undergraduates ( 42 males and 68 females) volunteered to participate in the study as a means of obtaining partial credit in an in- 
troductory psychology course. They took part in the study in groups of from 1 to 4 subjects.

\section{Materials}

The photographs were solicited from 12 married couples. The couples were selected so as to generate a set that was homogeneous with respect to physical characteristics that could have been used as judgmental cues revealing who might have been married to whom. Thus, all were of the same race, ethnic background, and age cohort. Separate full-face portraits of each partner taken sometime during their 1st year of marriage and separate portraits of each partner during approximately their 25 th year of marriage were obtained. The photographs were all of Caucasian couples who, at the time of the study, were 50 to 60 years of age. The couples resided either in the Detroit metropolitan area or in rural south-central Wisconsin.

Black-and-white $12.7 \times 17.8-\mathrm{cm}$ reproductions were made of the individual portraits. The faces were all enlarged to approximately the same size. Final prints were then cropped and masked with black $10.2 \times 11.4-\mathrm{cm}$ mats prepared with a $7.6-\mathrm{cm}$ ellipse-shaped window so that only the face and head of each person was visible. This procedure helped to conceal any extraneous features of the photographs, such as clothing or surround, that could have served as cues in resemblance and marriage likelihood judgments. Only 4 of the 24 face stimuli came from photographs in which the pair posed together. For the control group, the identical sets were used. However, the faces were whited out, leaving only the outline, the hair, and the background within the window.

\section{Procedure}

In the experimental conditions, two groups of subjects, each consisting of 37 participants, were given two different sets of photographs to judge, each judging one "young" $(Y)$ and one "old" $(O)$ subset. However, no individual judged spouses of the same couple photographed at two different times. Thus, one group received subset $\mathrm{AY}$ and $\mathrm{BO}$, while the second group judged subsets BY and AO, where $A$ is one set of six couples and $B$ is another. Half of the subjects saw the "young" subset first and the "old" second, whereas the reverse order was used for the remaining 37 subjects.

In the experimental conditions, subjects were randomly assigned to one of two treatments. Half of the subjects judged man-woman photograph pairs for resemblance, and half for the likelihood of the pair being married. In the control condition, comprising 36 subjects, all judgments were those for resemblance. 


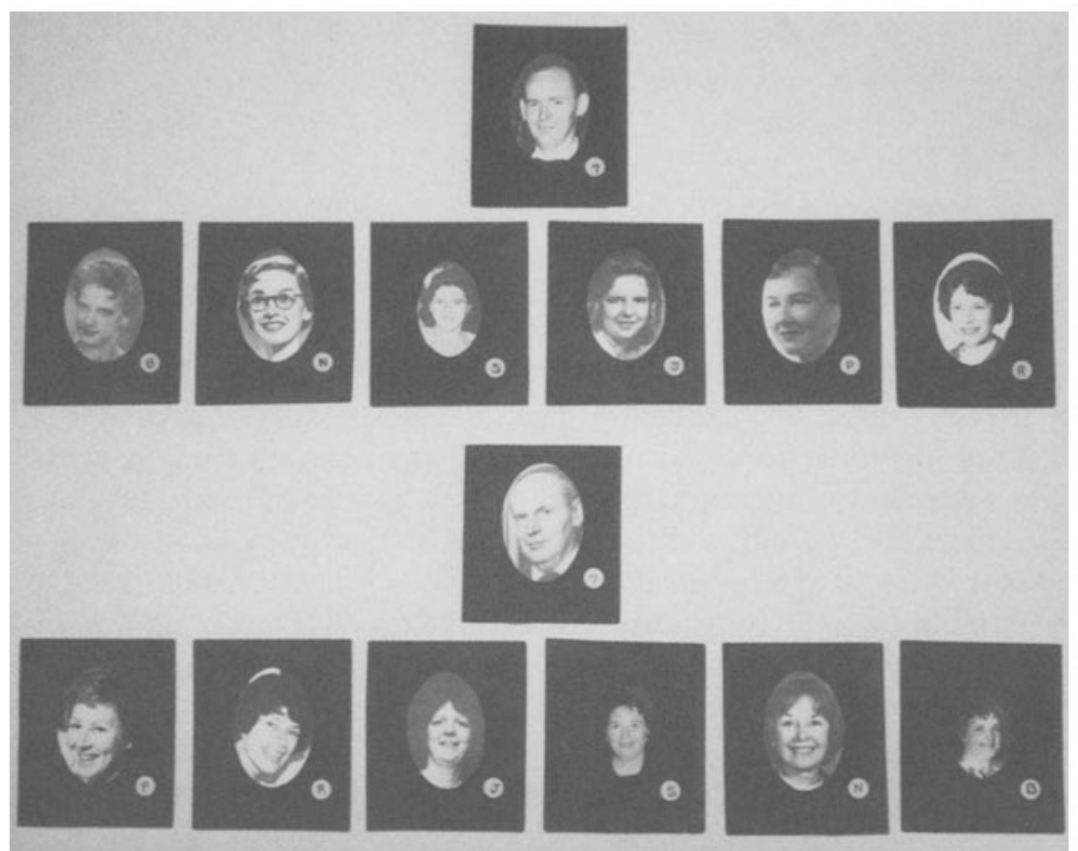

Fig. 1. Stimulus set $A Y$ (young) and AO (old), with a male target. The married couple is $7 \mathrm{P}$.

The photographs were displayed for judgment on $111.8 \times 71.1-\mathrm{cm}$ poster boards, outfitted with seven transparent plastic pockets. Six pockets were affixed in a single row about $2.5 \mathrm{~cm}$ apart. The seventh pocket was centered $28.5 \mathrm{~cm}$ below the row of six. On a given trial, a subset of same-sex photographs (AY, BY, AO, or BO) was displayed in the row of pockets, while the possible partners appeared, one a time, in the single "target" pocket. Figure 1 shows a typical "young" (top) stimulus set and its "old" counterpart (bottom). (Note, however, that different groups of subjects judged these two sets.) Half of the subjects were given wives as "targets" and half were given husbands.

Figure 2 shows the corresponding control stimuli. In the control group, only similarity judgments were obtained, however. Since this study addressed the question of growth of physiognomic similarity between spouses, that was the crucial variable, and if similarity could be detected on bases other than facial features, then no valid conclusions could be drawn about the major hypotheses.

Subjects noted their judgments in specially prepared booklets with pages designed to represent the layout of the display board. After general guide- 


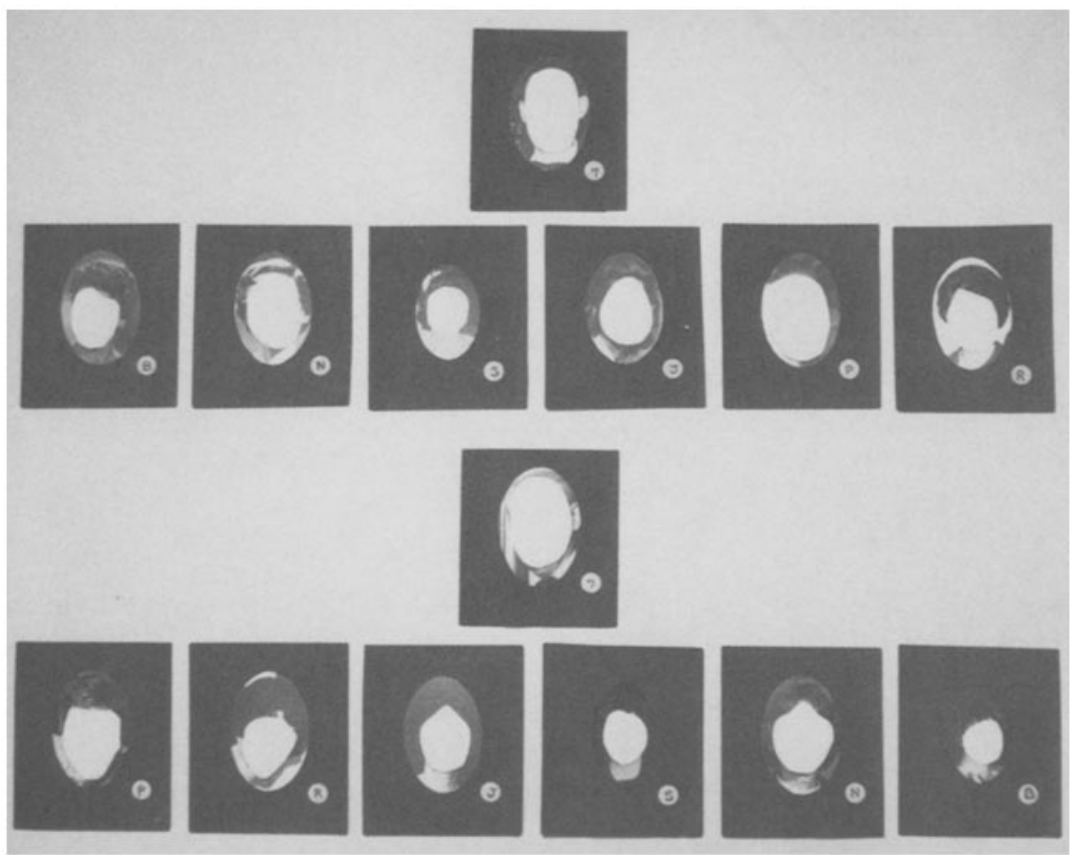

Fig. 2. Control stimuli for set $A Y$ (young) and $A O$ (old) couples, with a male target. The married couple is $7 \mathrm{P}$.

lines for the procedure that the subjects were to follow, the following instructions were given: "For this set of photographs, please write a number in each of the blanks underneath the boxes. Put a 1 under the photograph of the person who you think is the MOST LIKELY TO BE MARRIED to (who MOST CLOSELY RESEMBLES) the person in the target photo, a 2 under the photo of the person who is the next most likely candidate (next closest in resemblance), and so on."

The experimenter presented the photo board with the set of six comparison pictures and the target portrait for comparison. After subjects completed their rankings of the first target, the next target photo was inserted. The array in Figure 1 shows one target photograph and six photographs of the opposite sex, one of whom is the target's wife. Also shown is the same target and the same array of potential spouses 25 years later.

The procedure and instructions in the Control group were the same as in the Resemblance condition, except that subjects were warned about the possible difficulty of the task. The entire session (ranking 12 photos) lasted 
Table I. Mean Ranks of Marriage Likelihood and Resemblance Judgments for Married and Random Pairs, and Resemblance Judgments for the Control Group ${ }^{*}$

\begin{tabular}{lcccc}
\hline \multicolumn{1}{c}{ Condition } & Young & Old & $t$ & $p$ \\
\hline Married pairs & & & & \\
Marriage likelihood & 3.34 & 2.88 & 2.73 & .010 \\
Resemblance & 3.43 & 3.11 & 2.23 & .032 \\
Random pairs & & & & \\
$\quad$ Marriage likelihood & 3.45 & 3.59 & -.38 & .383 \\
$\quad$ Resemblance & 3.23 & 3.60 & -2.32 & .026 \\
Control group & & & & \\
$\quad$ Resemblance & 3.52 & 3.36 & .88 & .262 \\
\hline
\end{tabular}

${ }^{a}$ Because the table shows average ranks, lower values indicate greater resemblance and greater marriage likelihood.

from 15 to 20 minutes. After the session the subjects were thanked and debriefed.

\section{RESULTS}

The sex of the subject, the sex of the target, and the order of presentation ( $Y$ first vs. Y second) had no significant effects on the rankings. They were therefore eliminated from the main analysis. Table I shows the mean ranks assigned by subjects to each subset they judged. Differences between rankings for "young" versus "old" photographs were tested. In addition, random male-female pairs were created by selecting photographs at random, and the average ranks for these random couples were compared to the rankings for matching couples. Finally, the differences between the couples' (both matching and random) average ranks and the chance value of 3.5 were examined. The significant differences, therefore, that were obtained between the "young" and "old" photographs show that after 25 years the two spouses are perceived as more similar in appearance by the subjects, and that they are judged more likely to be married to each other. The differences found as a function of age seem to be somewhat more pronounced for the marriage than for the resemblance judgments, but this effect was not significant $(F(1,72)=.42)$.

On both similarity and marriage likelihood judgments, the average ranks of the "old" photographs departed significantly from the chance rank of 3.5. For marriage likelihood ratings, the average rank of 2.88 differed from 3.5 at the .001 level $(t(36)=4.83)$. For resemblance ratings, the average rank of 3.11 also differed from 3.5 at the .001 level $(t(36)=4.12)$.

The data on randomly selected "targets" showed no increase in either judged similarity or marriage likelihood with age. Moreover, three of the 
four random couples' values were not significantly different from the chance rank of 3.5. Hence, it can be concluded that for married couples the growth in perceived resemblance is not simply a reflection of an increased homogeneity of all older people. If that were the case, randomly paired older men and women would have been perceived more similar than randomly paired younger men and women. If anything, the data show the opposite tendency.

However, there is no tendency for the subjects to perceive the "young" married couples as more similar to each other than they would be by chance. We would expect such an effect from a propinquity theory of marriage (Burgess \& Wallin, 1953; Vandenberg, 1972). Neither on marriage ratings (mean rank $=3.34$ ) nor on resemblance ratings (mean rank $=3.43$ ) are the results reliably different from 3.5 , the mean rank we would expect by chance $(t(36)$ $=1.46$ and $t(36)=.68$, respectively).

The control group -i.e., subjects who viewed the photographs with the facial features obliterated-shows no effect. This result demonstrates that facial features are essential in arriving at a similarity judgment. Hence, background and other aspects of the photographs could not serve as cue in judging the males and females for resemblance.

\section{DISCUSSION}

Judgments of photographs of spouses taken at the time of their marriage and 25 years later show that spouses' resemblance to each other is greater after 25 years of marriage. This effect cannot be explained by the supposition that all people generally grow more alike as they grow old because randomly matched older couples do not appear more similar than randomly matched young couples. The greater perceived resemblance and marriage likelihood for photographs taken at the time of the spouses' silver wedding anniversary could not be due to judgment artifacts because no subject judged the same couple at both ages, and all age comparisons were made on a between-subject basis. Further, the control group data show that judgments must have been made on the basis of facial features alone. Thus, it appears from the present data that there may have been a real convergence in appearance for people who had been married to each other for two and a half decades.

Given that a convergence in physical appearance has been demonstrated, how can it be explained? One hypothesis offered in the epigraph as an illustration of a commonsense explanation suggests that the convergence of physical appearance may be due to the fact that the couple has shared the same diet over the course of many years. This hypothesis cannot be rejected offhand because most spouses do indeed share common diets. Perhaps diets that are similar in fat content might lead to similar amounts of fatty tissue 
in the face and thus increase the appearance of similarity. To explore the common diet hypothesis, 10 pilot subjects, recruited from the same pool as those who participated in the main study, were asked to rank-order the husbands and the wives from heaviest to lightest in weight. If, because of similar diets, husbands and wives converge in the amounts of fatty tissue and thus increase their phenotypic resemblance over time, we should find the rank correlations generated by the pilot subjects to be higher for the "old" photographs than for the "young" photographs. The opposite, however, was true. The average $r$ 's were .02 and -.24 , respectively, for the "young" and "old" sets - low in one group and in the wrong direction in the other. It is unlike$1 \mathrm{y}$, therefore, that facial fat was a significant cue in similarity judgments.

Another possibility is that because people who are married to each other live in the same region of the country, and consequently experience the same climate, they eventually grow to look alike. Thus, a married couple from Phoenix, suntanned and wrinkled by the Arizona weather, might look distinctive among couples from other regions. In the present study, however, all the couples judged came from the same midwestern region and represent the same socioeconomic level, age cohort, and other demographic characteristics that could make some couples stand out. Hence, geographic or demographic differences could not act as cues for matching spouses or for judging them similar.

A third possibility ${ }^{3}$ is that people who are predisposed to eventually look alike find each other as lovers and spouses. Given, for example, that both are disposed to a mid-age depression, they may not yet resemble each other at the time of their wedding but may eventually grow to look alike because they acquire depressive facial expressions. In principle, convergence in physical appearance caused by codiathesis could be subjected to a limited empirical test. One might, for example, predict that there is a physical convergence among spouses even if they had an early divorce. Such an empirical test, however, would be only partially informative because if convergence was not found, it could be argued that the marriage did not work out just because the partners were predisposed not to converge in physical appearance. Moreover, the hypothesis predicts that all depressives would eventually grow to look alike whether or not they were married to each other, and so would neurotics, hysterics, and Alzheimer's risks.

There is, however, one possible explanation that derives from a theory of emotional efference recently reclaimed (Zajonc, 1985). Eight decades ago, Waynbaum (1907) offered the hypothesis that expressions made in the course of an emotional process produce vascular changes because the facial muscles can act as ligatures on veins and arteries. He thought that the muscular 
actions of the face participate in maintaining and restoring cerebral blood flow because of the intimate relationship between arterial flow to the face and that to the brain (the main carotid artery is divided at the neck into the internal artery that supplies the brain and the external artery that supplies the face and tissues covering the skull). He also thought that changes in vascular equilibrium in the brain have hedonic effects, experienced by the individual as subjective feeling states such as sadness, joy, or excitation. Waynbaum's assumptions about the vascular flow were wrong in a number of ways (Fridlund \& Gilbert, 1985; Zajonc, 1985). But if correct in principle, then the process he proposed may occur because cerebral blood flow changes have specific neurotransmitter effects $-a$ fact that could not have been known then.

Waynbaum's theory leads to the implication that if facial musculature is capable of altering emotional states, repeated and habitual facial action may bring about a more permanent facial "look"-a look that might have some correlation with the individual's temperamental predisposition. Hence, the now largely abandoned physiognomic theories (e.g., Stanton, 1924) may find in Waynbaum's ideas a new source of support. There are marked individual differences in the facial skeletal structure and musculature. Some muscles are not universally present: The risorius Santorini, for example-a muscle that participates in producing the smile and the grin-is absent in a significant proportion of the population. If the ability to experience certain feeling states and moods varies, to some extent, with the configuration of facial musculature and skeletal structure, and if individuals develop correspondingly different patterns of facial muscular action, then the correlation between emotional disposition and physical appearance is no longer a mystery.

But why should two people who live together grow similar in their physical appearance? Waynbaum's theory also has another implication. The theory helps explain how we might be able to experience subjectively what another person is feeling. When we witness a person in pain or a person in terrible fright, it is not uncommon for us to reproduce, in a limited way, the person's facial expressions. Unconsciously and involuntarily induced mimicry, according to the vascular theory, should produce in the observer a subjective state that is similar to, although not as intense as, that of the target person. Even mild emotional expressions recorded on still photographs tend spontaneously to elicit these expressions in the subject viewing them (Dimberg, 1982).

Thus, the vascular theory of emotional efference suggests a process of empathy that is quite different from those postulated by other current theories (e.g., Hoffman, 1985). It implies that empathy is a process that relies on the motor engagement of the face and on the resulting subjective experience 
of a correlated feeling state. The person who empathizes with another can actually appreciate the other's condition because of his or her own subjective experience. And for this subjective experience to take place, nothing more is required than a matching facial expression.

People who reside in each other's immediate environment for long periods of time have frequent occasions to empathize with one another. A high frequency of repeated emotional expressions leaves marks on the face. Patients suffering long bouts of depression, for example, acquire a "depressed look." Similarly, repeated empathic responding to a marital partner may result in increased morphological similarity of the partners' faces, a phenomenon that has not escaped anecdotal observations such as those cited in the epigraph above.

In marriage, the partners typically remain in the same immediate environment and, because of the nature of their relationship, empathize with a variety of their spouses' emotional states. Over time, therefore, these various emotional states are shared, albeit in reduced intensity, such that a substantial proportion of the emotions of the wife are also experienced by the husband, and vice versa. Moreover, they are experienced in a similar sequence, in the same environmental context, and in a similar span of time. If the facial musculature plays a significant role in producing these emotional states and moods, then, with time, married partners should grow to resemble each other physically. Thus, for example, a couple that wins a lottery shares a joint positive emotional experience under the same circumstances and at about the same age. Likewise, parents who experience the death of child face a traumatic experience together at the same time. Mimicked expressions that are a daily occurrence would leave wrinkles around the mouth and eyes and would alter the bearing of the head and the overall expression. Eventually, they would produce morphological changes that make the spouses appear more similar than they originally were. These effects are confounded with the long-term effects of mimicry. It is difficult to estimate the extent to which a few strong emotional experiences shared by a couple would leave permanent identical marks on their faces. This study, however, attempted to address this question by means of self-report.

If empathy derives more directly from facial mimicry, and if facial motor mimicry is a central component of close human interactions, we should expect that couples who have grown more similar in physical appearance over time also have had a better marriage than couples who have grown only slightly more similar, or less similar, over a span of 25 years.

In order to address these questions, a mail survey of the 12 couples from whom the portraits were obtained was conducted. The survey asked respondents to supply information about their perceived similarity to their spouses at time of marriage and at present. The questionnaire also included 
items about each individual's level of satisfaction with the marriage and the number and kind of highly positive or highly negative experiences the couple shared. Each husband and wife was sent a questionnaire separately. Of the 24 married partners, 22 sent the questionnaires back. It was found that the greater the increase in resemblance of a couple over 25 years, the greater the couple's increase in self-reported happiness $(r=.49, p<.05)$, frequency of sharing worries and concerns $(r=.46, p<.05)$, and perceived similarity of attitudes $(r=.56, p<.01)$. However, the number and kind of shared experiences did not yield significant differences.

The results in Table I also indicate that when first married, spouses are not perceived as more similar to each other than would be expected by chance ( 3.43 vs. 3.50$)$. Nor are they perceived as more similar to each other than randomly paired couples ( 3.43 vs. 3.23 ). These results seem to contradict Spuhler's (1968) data and those of Rushton, Russell, and Wells (1985). The data on physical resemblance of spouses have been interpreted to indicate the type of assortative mating that is more likely to perpetuate each mate's genes. In a marriage of phenotypically similar individuals, the genes of each have a greater likelihood of being transmitted onto the offspring than in a marriage of unlike mates because the couple shares common genes. Note, however, that in the present study, the target persons were deliberately selected from a homogeneous population so that the growth of similarity between the spouses over time would not be obscured by much greater between-than within-couple variance. Perhaps phenotypic similarity of "young" mates could be better detected if the set of couples were more diverse and included both ethnic and racial differences. It might be, therefore, that within a homogeneous population the early physical similarity of mates may not be detectable.

Some important implications follow from the present research. The results obtained here suggest that the similarity among siblings, on the one hand, and between parents and offspring, on the other, may not be entirely a matter of common genes. Like married couples, siblings share a proximal environment and are vulnerable to long-term mimicry effects, and so are parents and their children. We should, therefore, expect adopted children, over time, to increase their resemblance to their foster parents and to foster siblings. Likewise, half-siblings, if they live under the same roof, would also eventually increase their resemblance to each other. Our results also suggest that spouses who were divorced early in marriage should look less similar when older than spouses who remained married during that period.

Furthermore, if judgments were to be made for the same couple over several successive intervals, a gradual increase in perceived resemblance should be obtained. Both of these last questions can be readily examined in studies using the present paradigm. If the process of facial efference, which is hypothesized to account for the growing resemblance of spouses, is indeed 
responsible for the physiognomic convergence of spouses, then it follows that phenotypic facial similarity within kinship groups is not simply a matter of common genes but can also develop as a consequence of prolonged social contact.

\section{REFERENCES}

Burgess, E. W., \& Wallin, P. (1953). Engagement and marriage. New York: Lippincott. Dimberg, U. (1982). Facial reactions to facial expressions. Psychophysiology, 19, 643-647. Fridlund, A. J., \& Gilbert, A. N. (1985). Emotions and facial expression. Science, 230, 607-608. Hoffman, M. L. (1985). Interaction of affect and cognition in empathy. In C. E. Izard, J. Kagan, \& R. B. Zajonc (Eds.), Emotion, cognition, and behavior. Cambridge: Cambridge University Press.

Rushton, J. P., Russell, R. J. H., \& Wells, P. A. (1985). Personality and genetic similarity theory. Journal of Social and Biological Structures, 8, 63-86.

Spuhler, J. N. (1968). Assortative mating with respect to physical characteristics. Eugenics Quarterly, $15,128-140$.

Stanton, M. (1924). The encyclopedia of face and form reading. Philadelphia: Davis.

Vandenberg, S. G. (1972). Assortative mating, or who marries whom? Behavior Genetics, 2, $127-157$.

Waynbaum, I. (1907). La physionomie humaine: Son mécanisme et son role social. Paris: Alcan.

Zajonc, R. B. (1985). Emotion and facial efference. Science, 228, 15-21. 